\title{
Philosophiques
}

\section{Henri Meschonnic, Le langage Heidegger, collecton Écriture, Paris, Presses Universitaires de France, 1990, 398 pages.}

\section{Marc Imbeault}

Volume 18, numéro 1, printemps 1991

URI : https://id.erudit.org/iderudit/027147ar

DOI : https://doi.org/10.7202/027147ar

Aller au sommaire du numéro

Éditeur(s)

Société de philosophie du Québec

ISSN

0316-2923 (imprimé)

1492-1391 (numérique)

Découvrir la revue

Citer ce compte rendu

Imbeault, M. (1991). Compte rendu de [Henri Meschonnic, Le langage

Heidegger, collecton Écriture, Paris, Presses Universitaires de France, 1990, 398

pages.] Philosophiques, 18(1), 182-184. https://doi.org/10.7202/027147ar d'utilisation que vous pouvez consulter en ligne.

https://apropos.erudit.org/fr/usagers/politique-dutilisation/ 
HENRI MESCHONNIC, Le langage Hetdegger, collection Écriture, Paris, Presses Universitaires de France, 1990, 398 pages.

par Marc Imbeault

Ce livre s'inscrit dans le mouvement de ressac qu'a provoqué en France la prise de conscience du sens de l'engagement politique de Martin Heidegger lors de la montée et de la chute du nazisme en Allemagne. Il est toutefois fort différent, par son objet (le langage), de celui de Farias par où le scandale a éclaté il y a quelque temps. C'est du point de vue de la poétique que Meschonnic tente d'élaborer une critique de la pensée heideggérienne. Selon l'auteur, le philosophe de Fribourg procède tout au long de son ceuvre et, bien sûr, tout spécialement dans ses commentaires de poèmes, à une *essentialisation * de la poésie qui se fait au détriment de son contenu.

L'expression * langage Heidegger * qui forme le titre de l'ouvrage demeure assez vague: * Le langage Heidegger - pas l'affaire ou le cas Heidegger, sinon dans la mesure de ce qu'ils disent du langage, et que la théorie peut en apprendre - est donc ce que j'entreprends d'analyser. Et il déborde Heidegger. Il inclut le langage de Heidegger, et un effet Heidegger. C'est ce que j'entends par le langage Heidegger. (20) L'auteur en parle souvent mais, presque immanquablement, c'est pour y ajouter un attribut nouveau. Heidegger serait à l'origine d'une nouvelle manière de lire et d'écrire en Occident. Et d'une manière de penser qui a contribué à la constitution d'une époque «non critique * (Ibid.). Les premiers chapitres contiennent par exemple des attaques virulentes à l'endroit du mimétisme des épigones parisiens de Heidegger. (Les amateurs d'insultes indirectes seront comblés.) Il est impossible de relater ici tous les aspects de cette critique du heideggérianisme. Ils concernent aussi bien le rapport du langage au poème et à l'éthique qu'à l'histoire et au politique. Je me contenterai, pour ma part, de discuter le rapport du langage au poème. C'est d'ailleurs, à mon avis, le domaine où l'auteur est le plus précis et le plus compétent ; sa critique du statut de l'histoire, en comparaison, m'a paru obscure.

L'auteur accuse Heidegger d'avoir rejeté d'un revers de main le travail des spécialistes du langage : linguistes, philologues et critiques littéraires. Il lui reproche entre autres d'avoir occulté le langage au profit d'une prétendue vérité du langage. Vérité dont le philosophe serait le dépositaire. C'est l'arbitraire qui gêne dans les propos de Heidegger sur la poésie. L'auteur appuie sa critique sur de nombreux exemples de commentaires de poèmes, de Hölderlin, Trakl, Rilke, Hebel et Char 
essentiellement. Heidegger aurait commis deux fautes principales. D'abord celle de détoumer honteusement les œuvres de ces poètes au profit de son entreprise de re-fondation de la métaphysique occidentale. L'idée que toute interprétation est une violence faite au texte servant à justifier une falsification pure et simple du contenu des poèmes. Mais la deuxième faute est encore plus grossière : lorsque Heidegger ne falsifie pas les poèmes, il se contente de les paraphraser. Quant aux heideggériens, non seulement ils acceptent de Heidegger ce que l'on $n$ 'accepterait pas d'un écolier, mais ils en remettent dans la paraphrase.

Meschonnic cite systématiquement le texte allemand de Heidegger après en avoir proposé une traduction. Il cite souvent en note d'autres traductions du même texte allemand. Le lecteur est ainsi amené à lire jusqu'à trois ou quatre fois la même citation (en allemand et dans des traductions différentes. Cette solution n'est certes pas économique - le livre pourrait compter sans cela cinquante ou soixante pages de moins - mais elle satisfera peut-être les heideggériens purs et durs qui croient encore que les faiblesses de la pensée de leur maître en français ne peuvent provenir que d'une erreur de traduction, ou encore de l'inadéquation congénitale de la langue française à l'expression de la pensée authentiquement philosophique.

Mais, en dépit de ces précautions, l'auteur n'est pas du tout partisan de l'idée selon laquelle il existerait des langues philosophiques privilégiées, en l'occurrence, selon l'orthodoxie heideggérienne, le grec et l'allemand. L'auteur y revient à plusieurs reprises, mais je ne relèverai qu'un seul aspect de son argumentation. D'après lui les tenants de la thèse heideggérienne seraient en réalité les défenseurs du truisme selon lequel : «allemand c'est allemand, grec c'est grec * (310). Les mots allemands ont sans doute un sens allemand et les mots français un sens français, mais ce truisme n'a de sens qu'en regard du mot isolé. Il en est tout autrement pour la langue et pour le discours comme système: « Pas-de-mot-pour est un argument ambigu et fallacieux, circulaire. Ainsi, selon Emmanuel Levinas, le judaïsme n'est pas une religion parce qu'il n'y a pas en hébreu de mot pour "religion.. Qu'aucun des mots du champ lexical du religieux n'y veut dire « religion *. En effet. Puisque c'est un terme du latin chrétien. Qui a un sens chrétien. Aucun des mots hébreux n'est ce latin-là. Ce qui ne signifie pas que quelque chose, non du mot, mais de la notion, n'en soit, autrement, présent." (Ibid.)

La thèse sur le caractère authentiquement philosophique de l'allemand par rapport aux autres langues vivantes, constitue un excellent repoussoir pour éviter d'avoir à expliquer la proverbiale intraduisibilité des cuvres de Heidegger. Ce qui devrait constituer un élément de suspicion à l'égard de cette ouvre, peut ainsi devenir la marque de la profondeur du penseur et de sa langue. Sans compter que cela a permis à toute une génération de germanistes francophones de dire : on ne peut comprendre la profondeur et la justesse de la pensée de Heidegger qu'en étant un expert de la langue et de la culture allemande, or nous sommes les dépositaires de cetre langue et de cette culture dans le monde francophone, donc nous sommes les seuls à comprendre ce dont il est question chez Heidegger. Moyennant quoi on pouvait toujours rétorquer aux rationalistes et autres dinosaures de la philosophie qui s'inquiétaient 
de l'obscurité de certains passage d'Être et Temps qu'ils ne comprenaient pas. Ce qui était vrai mais ne voulait pas dire qu'il y avait toujours quelque chose à comprendre, et surtout pas toujours quelque chose de profond.

Le langage Heidegger est somme toute un ouvrage assez stimulant. On peut lui reprocher d'être parfois superficiel, sans compter l'allure de règlement de compte que prennent certaines critiques à l'endroit des phénoménologues parisiens. Ces défauts sont toutefois en partie compensés par une plume alerte et un sens de la formule qui ne se dément pas du début à la fin. Bref, un livre qui choque parfois le lecteur avisé mais qui ne l'endort jamais.

Université de Paris I 